\title{
Some Properties and Regions of Variability of Affine Harmonic Mappings and Affine Biharmonic Mappings
}

\author{
Sh. Chen, ${ }^{1}$ S. Ponnusamy, ${ }^{2}$ and X. Wang ${ }^{1}$ \\ ${ }^{1}$ Department of Mathematics, Hunan Normal University, Changsha, Hunan 410081, China \\ ${ }^{2}$ Department of Mathematics, Indian Institute of Technology Madras, Chennai 600 036, India
}

Correspondence should be addressed to X. Wang, xtwang@hunnu.edu.cn

Received 28 September 2009; Accepted 21 December 2009

Recommended by Narendra Kumar Govil

We first obtain the relations of local univalency, convexity, and linear connectedness between analytic functions and their corresponding affine harmonic mappings. In addition, the paper deals with the regions of variability of values of affine harmonic and biharmonic mappings. The regions (their boundaries) are determined explicitly and the proofs rely on Schwarz lemma or subordination.

Copyright (c) 2009 Sh. Chen et al. This is an open access article distributed under the Creative Commons Attribution License, which permits unrestricted use, distribution, and reproduction in any medium, provided the original work is properly cited.

\section{Preliminaries and Main Results}

A planar harmonic mapping in a simply connected domain $D \subset \mathbb{C}$ is a complex-valued function $f=u+i v$ defined in $D$ such that $u$ and $v$ are real harmonic in $D$, that is, $\Delta u=0$ and $\Delta v=0$. Here $\Delta$ represents the complex Laplacian operator

$$
\Delta=4 \frac{\partial^{2}}{\partial z \partial \bar{z}}:=\frac{\partial^{2}}{\partial x^{2}}+\frac{\partial^{2}}{\partial y^{2}}
$$

The mapping $f$ has a canonical decomposition $f=h+\bar{g}$, where $h$ and $g$ are analytic (holomorphic) in $D[1,2]$. Lewy's theorem tells us that a harmonic mapping $f$ is locally univalent in $D$ if and only if its Jacobian $J_{f}(z) \neq 0$ for each $z \in D$ [3].

A four-time continuously differentiable complex-valued function $F=u+i v$ in $D$ is biharmonic if and only if the Laplacian of $F$ is harmonic. Note that $\Delta F$ is harmonic in $D$ if $F$ satisfies the biharmonic equation $\Delta(\Delta F)=0$. 
It is a simple exercise to see that every biharmonic mapping $F$ in a simply connected domain $D$ has the representation

$$
F=|z|^{2} G+K
$$

where $G$ and $K$ are some complex-valued harmonic functions in $D$. Recently, the class of biharmonic mappings has been studied by a number of authors [4-8]. It is known that a harmonic function of an analytic function is harmonic, but an analytic function of a harmonic function needs not be harmonic. Moreover, the only univalent harmonic mappings of $\mathbb{C}$ onto $\mathbb{C}$ are the affine mappings $g(z)=\beta z+\gamma \bar{z}+\eta$, where $|\beta| \neq|\gamma|$ (see [2, Section 2.4]).

We denote the class of analytic functions in the unit disk $\mathbb{D}=\{z \in \mathbb{C}:|z|<1\}$ by $\mathscr{H}$, and we think of $\mathscr{H}$ as a topological vector space endowed with the topology of uniform convergence over compact subsets of $\mathbb{D}$.

Definition 1.1. A function $F$ is said to be an affine harmonic mapping of $f \in \mathscr{H}$ if and only if

$$
F:=F_{\alpha}(f)=f+\alpha \bar{f}
$$

for some $\alpha \in \mathbb{C}$ with $|\alpha|<1$. If $f$ is biharmonic, then $F_{\alpha}(f)$ is called the affine biharmonic mapping of $f$.

It follows easily that every affine harmonic mapping is harmonic. Also, every affine biharmonic mapping is biharmonic. Definition 1.1 is motivated by the following result.

Theorem A (see [9, Theorem ]). For a harmonic mapping $f$, the following are equivalent:

(1) The Schwarzian derivative $S(f)=0$;

(2) $f=F_{\alpha}(h)$ for some (analytic) Möbius transformation $h$;

(3) $f$ takes circles to ellipses. defined by

Here $S(f)$ denotes the Schwarzian derivative of the harmonic mapping $f=h+\bar{g}$

$$
S(f)=2\left\{(\log \rho)_{z z}-\left((\log \rho)_{z}\right)^{2}\right\}, \quad \rho=\left|h^{\prime}\right|+\left|g^{\prime}\right|
$$

A domain $D \subset \mathbb{C}$ is said to be $M$-linearly connected if there exists a positive constant $M$ such that any two points $w_{1}, w_{2} \in D$ are joined by a path $\gamma \subset D$ with

$$
\ell(\gamma) \leq M\left|w_{1}-w_{2}\right|
$$

or equivalently

$$
\operatorname{diam}(\gamma) \leq M\left|w_{1}-w_{2}\right|
$$

where $\ell(\gamma)$ denotes the Euclidean length of $\gamma$ (cf. [10]). A complex-valued function $f$ is $M$ linearly connected if $f(\mathbb{D})$ is an $M$-linearly connected domain. 
In a recent paper, Chuaqui and Hernández [11] investigated the relation between the univalency of planar harmonic mappings $f$ and the linear connectedness of $f(\mathbb{D})$. They proved the following theorem.

Theorem B (see [11, Theorem 1]). Let $h: \mathbb{D} \rightarrow \mathbb{C}$ be a holomorphic univalent map. Then there exists a constant $c>0$ such that every harmonic mapping $f=h+\bar{g}$ with dilation $|\omega|<c$ is univalent in $\mathbb{D}$ if and only if $h(\mathbb{D})$ is an $M$-linearly connected domain, where $M$ can be taken to be $1 / c$.

We say that a complex-valued function $g$ defined on $\mathbb{D}$ is said to be convex in $\mathbb{D}$ if and only if $g(\mathbb{D})$ is a convex domain. A well-known analytic characterization gives that if $f$ is analytic in $\mathbb{D}$, then $f$ is convex in $\mathbb{D}$ if and only if

$$
\operatorname{Re}\left(1+z \frac{f^{\prime \prime}(z)}{f^{\prime}(z)}\right)>0, \quad z \in \mathbb{D}
$$

The main of this paper is twofold. We first discuss the close relationships that exist among the local univalency, convexity, and linear connectedness of $f$ and its corresponding $F_{\alpha}(f)$. One of our main results follows.

Lemma 1.2. Let $f \in \mathscr{H}$. Then

(1) $F_{\alpha}(f)$ is locally univalent if and only if $f$ is locally univalent;

(2) $F_{\alpha}(f)$ is convex if and only if $f$ is convex, where $f$ is locally univalent;

(3) $F_{\alpha}(f)$ is $M_{2}$-linearly connected if and only if $f$ is $M_{1}$-linearly connected, where $f$ is univalent and the constants $M_{1}$ and $M_{2}$ depend only on each other and $\alpha$.

Let $\mathcal{S}^{0}$ denote the class of analytic functions $f$ in $\mathbb{D}$ with $f(0)=0$, and

$$
\mathcal{S}_{1}^{0}=\left\{f \in \mathcal{S}^{0}:|\operatorname{Re}(f)|<1\right\}
$$

A function $f \in \mathscr{H}$ is called a Bloch function if

$$
\|f\|_{\mathcal{B}}=\sup _{z \in \mathbb{D}}\left(1-|z|^{2}\right)\left|f^{\prime}(z)\right|<\infty .
$$

Then the set of all Bloch functions form a complex Banach space $\mathbb{B}$ with the norm

$$
|f(0)|+\|f\|_{\mathcal{B}^{\prime}}
$$

see [12]. The following result shows that $\mathcal{S}_{1}^{0} \subset \boldsymbol{B}$.

Proposition 1.3. If $f \in S_{1}^{0}$, then $\|f\|_{\mathcal{B}} \leq 4 / \pi$. The constant $4 / \pi$ is sharp.

Proof. Let $f \in \mathcal{S}_{1}^{0}$ and let $P$ be defined by $P=g \circ h \circ f$, where

$$
g(z)=\frac{1-z}{1+z}, \quad h(z)=\exp \left(\left(\frac{i \pi}{2}\right) z\right)
$$


It follows that $P(z)=i \tan ((\pi / 4) f(z))$ and $P$ is analytic mapping of the unit disk $\mathbb{D}$ to $\mathbb{D}$ with

$$
P^{\prime}(z)=\frac{i \pi}{4}\left(1-P^{2}(z)\right) f^{\prime}(z), \quad z \in \mathbb{D}
$$

Also, by the classical Schwarz-Pick lemma, we have

$$
\left(1-|z|^{2}\right)\left|P^{\prime}(z)\right| \leq 1-|P(z)|^{2}, \quad z \in \mathbb{D}
$$

which gives

$$
\frac{\pi}{4}\left(1-|z|^{2}\right)\left|1-P^{2}(z)\right|\left|f^{\prime}(z)\right| \leq 1-|P(z)|^{2},
$$

whence

$$
\left(1-|z|^{2}\right)\left|f^{\prime}(z)\right| \leq \frac{4}{\pi} \frac{1-|P(z)|^{2}}{\left|1-P(z)^{2}\right|} \leq \frac{4}{\pi} .
$$

Thus, we have $\|f\|_{B} \leq 4 / \pi$.

For the proof of sharpness part, we consider

$$
f(z)=-\frac{2 i}{\pi} \log \left(\frac{1+e^{i \alpha} z}{1-e^{i \alpha} z}\right), \quad \alpha \in \mathbb{R}
$$

Then $f \in \mathcal{S}^{0}$ with $|\operatorname{Re}(f(z))|<1$ for $z \in \mathbb{D}$ and

$$
f^{\prime}(z)=-i \frac{4}{\pi}\left(\frac{e^{i \alpha}}{1-e^{2 i \alpha} z^{2}}\right),
$$

which gives $\|f\|_{\mathcal{B}}=4 / \pi$. The proof is complete.

Let $S_{u}=\left\{f: f \in S_{1}^{0}\right.$ and $f$ is univalent $\} \cup\{0\}$.

For any fixed $z_{0} \in \mathbb{D} \backslash\{0\}$ and $\lambda \in \mathbb{C}$ with $0<|\lambda|<1$, we define

$$
\begin{aligned}
V\left(z_{0}\right) & =\left\{f\left(z_{0}\right): f \in \mathcal{S}_{u}\right\}, \\
V_{\mathscr{H}}\left(z_{0}\right) & =\left\{F_{\alpha}(f)\left(z_{0}\right): f \in \mathcal{S}_{u}\right\}, \\
V\left(z_{0}, \lambda\right) & =\left\{f\left(z_{0}\right): f \in \mathcal{S}_{1}^{0}, f^{\prime}(0)=\frac{4 \lambda}{\pi}\right\}, \\
V_{\mathscr{H}}\left(z_{0}, \lambda\right) & =\left\{F_{\alpha}(f)\left(z_{0}\right): f \in \mathcal{S}_{1}^{0}, f^{\prime}(0)=\frac{4 \lambda}{\pi}\right\} .
\end{aligned}
$$


Let $B \mathscr{\ell}$ denote the class of all functions $f$ that are biharmonic but not harmonic in $\mathbb{D}$ with the normalization $f_{\bar{z}}(0)=1$ and $|f(z)| \leq 1$. For any fixed $z_{0} \in \mathbb{D}$, let

$$
V_{B \mathscr{H}}\left(z_{0}\right)=\left\{F_{\alpha}(f)\left(z_{0}\right): f \in ß \mathscr{H}\right\} .
$$

Recently, by using the Herglotz representation formula for analytic functions, Yanagihara [13], and later in a number of papers Ponnusamy and Vasudevarao (see, e.g., [14]), discussed region of variability problems for a number of classical subclasses of univalent and analytic functions in the unit disk $\mathbb{D}$. Because the class of harmonic univalent mappings includes the class of conformal mappings, it is natural to study the class of harmonic mappings and the class of biharmonic mappings. In this paper, we also discuss the region of variability problems for the class of affine harmonic mappings and affine biharmonic mappings, respectively. By a different method of proof, we will also determine the regions of variability for $V_{\mathscr{H}}\left(z_{0}\right), V_{\mathscr{H}}\left(z_{0}, \lambda\right)$, and $V_{\bar{B} \mathscr{\ell}}\left(z_{0}\right)$. The following are our results.

Theorem 1.4. The boundary $\partial V_{d e}\left(z_{0}\right)$ of $V_{d e}\left(z_{0}\right)$ is the Jordan curve given by

$$
(-\pi, \pi] \ni \theta \longmapsto-i \frac{2}{\pi}\left(\log \frac{1+e^{i \theta} z_{0}}{1-e^{i \theta} z_{0}}+\alpha \overline{\log \frac{1+e^{i \theta} z_{0}}{1-e^{i \theta} z_{0}}}\right)
$$

Theorem 1.5. The boundary $\partial V_{d e}\left(z_{0}, \lambda\right)$ of $V_{d e}\left(z_{0}, \lambda\right)$ is the Jordan curve given by

$$
(-\pi, \pi] \ni \theta \longmapsto-i \frac{2}{\pi}\left(\log \frac{1+z_{0} i \delta\left(e^{i \theta} z_{0}, \lambda\right)}{1-z_{0} i \delta\left(e^{i \theta} z_{0}, \lambda\right)}+\alpha \overline{\log \frac{1+z_{0} i \delta\left(e^{i \theta} z_{0}, \lambda\right)}{1-z_{0} i \delta\left(e^{i \theta} z_{0}, \lambda\right)}}\right)
$$

where

$$
\delta\left(e^{i \theta} z, \lambda\right)=\frac{e^{i \theta} z+\lambda}{1+e^{i \theta} z \bar{\lambda}}
$$

The following two lemmas are useful for the proofs of Theorems 1.4 and 1.5.

Lemma 1.6. Both sets $V\left(z_{0}\right)$ and $V_{d e}\left(z_{0}\right)$ are compact and convex.

Proof. Obviously, both sets $V\left(z_{0}\right)$ and $V_{\mathscr{l}}\left(z_{0}\right)$ are convex. Since $\mathcal{S}_{u}$ is closed, the compactness of sets $V\left(z_{0}\right)$ and $V_{\mathscr{d}}\left(z_{0}\right)$ easily follows from Proposition 1.3.

The argument as in the proof of Lemma 1.6 yields the following.

Lemma 1.7. Both sets $V\left(z_{0}, \lambda\right)$ and $V_{d e}\left(z_{0}, \lambda\right)$ are compact and convex.

Finally, we have

Theorem 1.8. The boundary $\partial V_{B \mathcal{B}}\left(z_{0}\right)$ of $V_{B \mathcal{H}}\left(z_{0}\right)$ is an ellipse.

Proofs of Lemma 1.2, and Theorems 1.4, 1.5, and 1.8 will be presented in Section 2. 


\section{Proofs of Main Results}

Proof of Lemma 1.2. (1) We see that $J_{F}=\left(1-|\alpha|^{2}\right) J_{f}$ and the proof of Lemma 1.2(1) follows.

(2) Let $f$ be analytic and locally univalent in $\mathbb{D}$, and $z=r e^{i t}$, where $r \in(0,1)$ and $t \in[0,2 \pi)$. Then for any fixed $r \in(0,1)$,

$$
\begin{aligned}
\frac{d F_{\alpha}(f)\left(r e^{i t}\right)}{d t} & =i r e^{i t} f^{\prime}\left(r e^{i t}\right)-i \alpha r e^{-i t} \overline{f^{\prime}\left(r e^{i t}\right)} \\
& =i\left(z f^{\prime}(z)-\alpha \overline{z f^{\prime}(z)}\right) .
\end{aligned}
$$

Since the curvature of $F_{\alpha}(f)$ is defined by

$$
\mathcal{K}_{F_{\alpha}(f)}=\frac{d\left(\arg \left(d F_{\alpha}(f) / d t\right)\right)}{d s}=\frac{d\left(\arg \left(d F_{\alpha}(f) / d t\right)\right)}{d t} / \frac{d s}{d t}
$$

we see that

$$
\begin{aligned}
\left|\frac{d F_{\alpha}(f)}{d t}\right| \mathcal{K}_{F_{\alpha}(f)} & =\frac{d}{d t}\left(\arg \frac{d F_{\alpha}(f)\left(r e^{i t}\right)}{d t}\right) \\
& =\operatorname{Re}\left(\frac{z f^{\prime}+z^{2} f^{\prime \prime}+\alpha \overline{z f^{\prime}}+\alpha \overline{z^{2} f^{\prime \prime}}}{z f^{\prime}-\alpha \overline{z f^{\prime}}}\right) .
\end{aligned}
$$

Multiplying both sides by

$$
\left|z f^{\prime}(z)-\alpha \overline{z f^{\prime}(z)}\right|^{2}=\left(z f^{\prime}(z)-\alpha \overline{z f^{\prime}(z)}\right)\left(\overline{z f^{\prime}(z)}-\bar{\alpha} z f^{\prime}(z)\right)
$$

and then simplifying the resulting expression on the right give

$$
\frac{1}{|z|^{2}}\left|\frac{d F_{\alpha}(f)}{d t}\right|^{3} \mathcal{K}_{F_{\alpha}(f)}=\left|f^{\prime}(z)\right|^{2}\left(1-|\alpha|^{2}\right) \operatorname{Re}\left(1+\frac{z f^{\prime \prime}(z)}{f^{\prime}(z)}\right) \text {. }
$$

The proof of Lemma Lemma 1.2(2) follows from (1.7).

(3) For the proof of the sufficiency, let $f(\mathbb{D})$ be $M_{1}$-linearly connected and

$$
H(w)=F_{\alpha}(f)\left(f^{-1}(w)\right)=w+\alpha \bar{w}
$$

where $w \in f(\mathbb{D})$. For any distinct points $w_{1}, w_{2} \in f(\mathbb{D})$, there exists a path $\gamma \subset f(\mathbb{D})$ joining $w_{1}$ and $w_{2}$ such that

$$
\ell(\gamma) \leq M_{1}\left|w_{1}-w_{2}\right|
$$


Let $\Gamma=F_{\alpha}(f)(\gamma)$. Then we have

$$
\begin{aligned}
\ell(\Gamma) & =\int_{\Gamma}|d H(w)| \\
& =\int_{\gamma}\left|H_{w} d w+H_{\bar{w}} d \bar{w}\right| \\
& \leq \ell(\gamma)(1+|\alpha|) \\
& \leq(1+|\alpha|) M_{1}\left|w_{1}-w_{2}\right| .
\end{aligned}
$$

On the other hand,

$$
\left|H\left(w_{1}\right)-H\left(w_{2}\right)\right|=\left|w_{1}+\alpha \overline{w_{1}}-w_{2}-\alpha \overline{w_{2}}\right| \geq(1-|\alpha|)\left|w_{1}-w_{2}\right| .
$$

Hence

$$
\ell(\Gamma) \leq \frac{M_{1}(1+|\alpha|)}{1-|\alpha|}\left|H\left(w_{1}\right)-H\left(w_{2}\right)\right|
$$

For the proof of necessity, since $F_{\alpha}(f)$ is univalent, we define

$$
G=f \circ F_{\alpha}(f)^{-1} .
$$

By the chain rule, we have

$$
G_{w}=f^{\prime} \cdot\left(F_{\alpha}(f)^{-1}\right)_{w^{\prime}} \quad G_{\bar{w}}=f^{\prime} \cdot\left(F_{\alpha}(f)^{-1}\right)_{\bar{w}} .
$$

Differentiating both sides of the equation, $F_{\alpha}(f)^{-1}\left(F_{\alpha}(f)(z)\right)=z$ yields the following relations:

$$
\begin{aligned}
& \left(F_{\alpha}(f)^{-1}\right)_{w} \cdot f^{\prime}+\bar{\alpha}\left(F_{\alpha}(f)^{-1}\right)_{\bar{w}} \cdot f^{\prime}=1 \\
& \alpha\left(F_{\alpha}(f)^{-1}\right)_{w} \cdot \overline{f^{\prime}}+\left(F_{\alpha}(f)^{-1}\right)_{\bar{w}} \cdot \overline{f^{\prime}}=0 .
\end{aligned}
$$

Solving these two equations yields

$$
\left(F_{\alpha}(f)^{-1}\right)_{w}=\frac{1}{\left(1-|\alpha|^{2}\right) f^{\prime}}, \quad\left(F_{\alpha}(f)^{-1}\right)_{\bar{w}}=-\frac{\alpha}{\left(1-|\alpha|^{2}\right) f^{\prime}}
$$

It follows from (2.12) that

$$
\left|G_{w}\right|+\left|G_{\bar{w}}\right|=\frac{1}{1-|\alpha|} .
$$


For any two distinct points $w_{1}, w_{2} \in F_{\alpha}(f)(\mathbb{D})$, since $F_{\alpha}(f)(\mathbb{D})$ is $M_{2}$-linearly connected, there exists a path $\gamma \subset F_{\alpha}(f)(\mathbb{D})$ joining $w_{1}$ and $w_{2}$ such that

$$
\ell(\gamma) \leq M_{2}\left|w_{1}-w_{2}\right|
$$

Now, we let $\Gamma=G(\gamma)$. Then we see that

$$
\begin{aligned}
\ell(\Gamma) & =\int_{\Gamma}|d G(w)| \\
& =\int_{\gamma}\left|G_{w} d w+G_{\bar{w}} d \bar{w}\right| \\
& \leq \frac{M_{2}\left|w_{1}-w_{2}\right|}{1-|\alpha|} .
\end{aligned}
$$

On the other hand, by the definition of $G$, we have

$$
w_{1}-w_{2}=G\left(w_{1}\right)-G\left(w_{2}\right)+\alpha\left(\overline{G\left(w_{1}\right)}-\overline{G\left(w_{2}\right)}\right)
$$

from which we obtain

$$
\left|w_{1}-w_{2}\right| \leq\left|G\left(w_{1}\right)-G\left(w_{2}\right)\right|(1+|\alpha|)
$$

and therefore,

$$
\ell(\Gamma) \leq \frac{M_{2}(1+|\alpha|)\left|G\left(w_{1}\right)-G\left(w_{2}\right)\right|}{1-|\alpha|} .
$$

The proof of the theorem is complete.

Before we indicate the proof of Theorem 1.4, we need to determine the region of variability $V\left(z_{0}\right)$.

Lemma 2.1. The boundary $\partial V\left(z_{0}\right)$ of $V\left(z_{0}\right)$ is the Jordan curve given by

$$
(-\pi, \pi] \ni \theta \longmapsto-\frac{2 i}{\pi} \log \left(\frac{1+e^{i \theta} z_{0}}{1-e^{i \theta} z_{0}}\right)
$$

Proof. For $f \in \mathcal{S}_{u}$, let $P=i \tan ((\pi / 4) f)$. Then $P(\mathbb{D}) \subset \mathbb{D}$. Using the Schwarz lemma, we have $|P(z)| \leq|z|$. That is,

$$
\left|\exp \left(\frac{\pi}{2} i f(z)\right)-1\right| \leq|z|\left|\exp \left(\frac{\pi}{2} i f(z)\right)+1\right|
$$


or equivalently

$$
\left|\exp \left(\frac{\pi}{2} i f(z)\right)-\frac{1+|z|^{2}}{1-|z|^{2}}\right| \leq \frac{2|z|}{1-|z|^{2}} .
$$

This yields that for each $z_{0} \in \mathbb{D}$,

$$
\exp \left(\frac{\pi}{2} i f\left(z_{0}\right)\right) \in \overline{\mathbb{D}}\left(C\left(z_{0}\right), R\left(z_{0}\right)\right)=\left\{w \in \mathbb{C}:\left|w-C\left(z_{0}\right)\right| \leq R\left(z_{0}\right)\right\}
$$

where

$$
C\left(z_{0}\right)=\frac{1+\left|z_{0}\right|^{2}}{1-\left|z_{0}\right|^{2}}, \quad R\left(z_{0}\right)=\frac{2\left|z_{0}\right|}{1-\left|z_{0}\right|^{2}}
$$

Consider

$$
f_{z_{0}}(z)=-\frac{2 i}{\pi} \log \frac{1+z_{0} z}{1-z_{0} z}
$$

Then $f_{z_{0}} \in \mathcal{S}_{u}$ and, for $z_{0} \in \mathbb{D} \backslash\{0\}$, the function $f_{z_{0}}$ is a nonconstant analytic function in $\mathbb{D}$. Hence it is an open mapping. Thus $f_{z_{0}}(\mathbb{D}) \subset V\left(z_{0}\right)$. Next we will show that $f_{z_{0}}(z) \in \partial V\left(z_{0}\right)$ for all $z \in \partial \mathbb{D}$. An elementary computation shows that

$$
\begin{aligned}
\exp \left(\frac{\pi}{2} i f_{z_{0}}(z)\right)-\frac{1+\left|z_{0}\right|^{2}}{1-\left|z_{0}\right|^{2}} & =\frac{1+z z_{0}}{1-z z_{0}}-\frac{1+\left|z_{0}\right|^{2}}{1-\left|z_{0}\right|^{2}} \\
& =\frac{1}{z}\left(\frac{z-\overline{z_{0}}}{\bar{z}-z_{0}}\right) \frac{2 z_{0}}{1-\left|z_{0}\right|^{2}},
\end{aligned}
$$

where $|z|=1$. Hence

$$
\exp \left(\frac{\pi}{2} i f_{z_{0}}(z)\right) \in \partial \mathbb{D}\left(C\left(z_{0}\right), R\left(z_{0}\right)\right)
$$

Since

$$
\begin{gathered}
\exp \left(\frac{\pi}{2} i f_{z_{0}}(z)\right) \in \exp \left(\frac{i \pi}{2} V\left(z_{0}\right)\right) \subset \overline{\mathbb{D}}\left(C\left(z_{0}\right), R\left(z_{0}\right)\right), \\
\exp \left(\frac{\pi}{2} i V\left(z_{0}\right)\right)=\left\{\exp \left(\frac{\pi}{2} i \xi\right): \xi \in V\left(z_{0}\right)\right\},
\end{gathered}
$$

we have

$$
\exp \left(\frac{\pi}{2} i f_{z_{0}}(z)\right) \in \partial \exp \left(\frac{i \pi}{2} V\left(z_{0}\right)\right)
$$


provided $z \in \partial \mathbb{D}$. Obviously, $G=\exp \left((\pi / 2) i f_{z_{0}}\right)$ is univalent in $\partial \mathbb{D}$. By Lemma 1.6, we know that $\partial \exp \left((i \pi / 2) V\left(z_{0}\right)\right)$ is a simple closed curve. Therefore, the boundary $\partial V\left(z_{0}\right)$ is the Jordan curve given by (2.21). The proof of the lemma is complete.

Proof of Theorem 1.4. The proof of Theorem 1.4 is an immediate consequence of Lemmas 1.2, 1.6, and 2.1. $V\left(z_{0}, \lambda\right)$.

Clearly, for the proof of Theorem 1.5, it suffices to describe the region of variability

Lemma 2.2. The boundary $\partial V\left(z_{0}, \lambda\right)$ of $V\left(z_{0}, \lambda\right)$ is the Jordan curve given by

$$
(-\pi, \pi] \ni \theta \longmapsto-\frac{2 i}{\pi} \log \left(\frac{1+z_{0} i \delta\left(e^{i \theta} z_{0}, \lambda\right)}{1-z_{0} i \delta\left(e^{i \theta} z_{0}, \lambda\right)}\right),
$$

where

$$
\delta(c z, \lambda)=\frac{c z+\lambda}{1+c z \bar{\lambda}}
$$

Proof. Let $f \in S_{1}^{0}$ with $f^{\prime}(0)=4 \lambda / \pi$. It follows from the classical Schwarz lemma for analytic functions that

$$
\left|\frac{\tan ((\pi / 4) f(z)) / z-\lambda}{1-\bar{\lambda}(\tan ((\pi / 4) f(z)) / z)}\right| \leq|z|
$$

which, after some computation, is equivalent to

$$
\left|\frac{e^{(\pi / 2) i f(z)}-A(z, \lambda)}{e^{(\pi / 2) i f(z)}+B(z, \lambda)}\right| \leq|z||T(z, \lambda)|
$$

where

$$
A(z, \lambda)=\frac{1+i \lambda z}{1-i \lambda z}, \quad B(z, \lambda)=\frac{\bar{\lambda}+i z}{i z-\bar{\lambda}^{\prime}}, \quad T(z, \lambda)=\frac{i z-\bar{\lambda}}{1-i \lambda z} .
$$

According to the Schwarz lemma, we see that the equality sign occurs in (2.33) if and only if $f=Q_{e^{i \theta}, \lambda}$ for some $\theta \in \mathbb{R}$, where

$$
Q_{c, \lambda}(z)=-\frac{2 i}{\pi} \log \frac{1+z i \delta(c z, \lambda)}{1-z i \delta(c z, \lambda)} .
$$

Further calculations show that the inequality (2.34) is equivalent to

$$
\left|\exp \left(\frac{\pi}{2} i f(z)\right)-C(z, \lambda)\right| \leq r(z, \lambda),
$$


where

$$
\begin{aligned}
C(z, \lambda) & =\frac{A(z, \lambda)+|z|^{2}|T(z, \lambda)|^{2} B(z, \lambda)}{1-|z|^{2}|T(z, \lambda)|^{2}}, \\
r(z, \lambda) & =\frac{|z||T(z, \lambda)||A(z, \lambda)+B(z, \lambda)|}{1-|z|^{2}|T(z, \lambda)|^{2}} .
\end{aligned}
$$

It follows from (2.35) that

$$
\begin{gathered}
1-|z|^{2}|T(z, \lambda)|^{2}=\frac{\left(1-|z|^{2}\right)\left[1+|z|^{2}-2 \operatorname{Re}(i \lambda z)\right]}{|1-i \lambda z|^{2}}, \\
A(z, \lambda)+B(z, \lambda)=\frac{2 i z\left(1-|\lambda|^{2}\right)}{(1-i \lambda z)(i z-\bar{\lambda})}
\end{gathered}
$$

so that $r(z, \lambda)$ simplifies to the form

$$
r(z, \lambda)=\frac{2|z|^{2}\left(1-|\lambda|^{2}\right)}{|1-i \lambda z|^{2}\left(1-|z|^{2}|T(z, \lambda)|^{2}\right)} .
$$

Further,

$$
A(z, \lambda)+|z|^{2}|T(z, \lambda)|^{2} B(z, \lambda)=\frac{|z|^{4}-2|\lambda z|^{2}+1+2 i\left(1-|z|^{2}\right) \operatorname{Re}(\lambda z)}{|1-i \lambda z|^{2}} .
$$

From (2.37), we have

$$
\exp \left(\frac{i \pi}{2} V\left(z_{0}, \lambda\right)\right) \subset \overline{\mathbb{D}}\left(C\left(\lambda, z_{0}\right), r\left(\lambda, z_{0}\right)\right) \triangleq\left\{w \in \mathbb{C}:\left|w-C\left(\lambda, z_{0}\right)\right| \leq r\left(\lambda, z_{0}\right)\right\}
$$

Equation (2.36) gives

$$
\exp \left(\frac{\pi}{2} i Q_{c, \lambda}(z)\right)=\frac{1+z\left(\bar{\lambda}_{c}+i \lambda\right)+c i z^{2}}{1+z\left(\bar{\lambda}_{c}-i \lambda\right)-c i z^{2}}
$$


and so, we infer from (2.35) that

$$
\begin{aligned}
& \exp \left(\frac{\pi}{2} i Q_{c, \lambda}(z)\right)-A(z, \lambda)=\frac{2 i c z^{2}\left(1-|\lambda|^{2}\right)}{(1-z i \lambda)\left[1+z\left(\bar{\lambda}_{c}-i \lambda\right)-c i z^{2}\right]^{2}} \\
& \exp \left(\frac{\pi}{2} i Q_{c, \lambda}(z)\right)+B(z, \lambda)=\frac{2 i z\left(1-|\lambda|^{2}\right)}{(z i-\bar{\lambda})\left[1+z\left(\bar{\lambda}_{c}-i \lambda\right)-c i z^{2}\right]}
\end{aligned}
$$

As

$$
w-C(z, \lambda)=\frac{1}{1-|z|^{2}|T(z, \lambda)|^{2}}\left\{(w-A(z, \lambda))-|z|^{2}|T(z, \lambda)|^{2}(w+B(z, \lambda))\right\}
$$

the above equalities together with (2.39), (2.40), and (2.42) yield that

$$
\exp \left(\frac{\pi}{2} i Q_{c, \lambda}(z)\right)-C(z, \lambda)=\frac{2 i z\left(1-|\lambda|^{2}\right)\left[c z+i c \bar{\lambda}|z|^{2}+\lambda|z|^{2}+i \bar{z}|z|^{2}\right]}{\left[1+z(c \bar{\lambda}-i \lambda)-i c z^{2}\right]|1-z i \lambda|^{2}\left(1-|z|^{2}|T(z, \lambda)|^{2}\right)}
$$

Substituting $c$ by $e^{i \theta}$ in the last equality, we see from (2.41) that

$$
\left|\exp \left(\frac{\pi}{2} i Q_{e^{i \theta, \lambda}}(z)\right)-C(z, \lambda)\right|=r(z, \lambda) \frac{\left|1+\bar{z}\left(e^{-i \theta} \lambda+i \bar{\lambda}\right)+i e^{-i \theta} \bar{z}^{2}\right|}{\left|1+z\left(e^{i \theta} \bar{\lambda}-i \lambda\right)-i e^{i \theta} z^{2}\right|}
$$

which implies that

$$
\exp \left(\frac{\pi}{2} i Q_{e^{i \theta, \lambda}}\left(z_{0}\right)\right) \in \exp \left(\frac{i \pi}{2} V\left(z_{0}, \lambda\right)\right) \subset \overline{\mathbb{D}}\left(C\left(\lambda, z_{0}\right), r\left(\lambda, z_{0}\right)\right)
$$

whence

$$
\exp \left(\frac{\pi}{2} i Q_{e^{i \theta, \lambda}}\left(z_{0}\right)\right) \in \partial \exp \left(\frac{i \pi}{2} V\left(z_{0}, \lambda\right)\right)
$$

We claim that the closed curve

$$
(-\pi, \pi] \ni \theta \longmapsto \exp \left(\frac{\pi}{2} i Q_{e^{i \theta, \lambda}}\left(z_{0}\right)\right)
$$

is simple. 

that

For the proof of this claim, we suppose that there are $\theta_{1}, \theta_{2} \in(-\pi, \pi]$ with $\theta_{1} \neq \theta_{2}$ such

$$
\exp \left(\frac{\pi}{2} i Q_{e^{i \theta_{1}, \lambda}}\left(z_{0}\right)\right)=\exp \left(\frac{\pi}{2} i Q_{e^{i \theta_{2}, \lambda}}\left(z_{0}\right)\right)
$$

By (2.36), we obtain that $\theta_{1}=\theta_{2}$ which is a contradiction and this completes the proof of our claim.

Since $V\left(z_{0}, \lambda\right)$ is a compact convex subset of $\mathbb{C}$ and its interior is nonempty, we see that its boundary $\partial V\left(z_{0}, \lambda\right)$ is a simple closed curve. It follows from (2.40) that the curve

$$
(-\pi, \pi] \ni \theta \longmapsto \exp \left(\frac{\pi}{2} i Q_{e^{i \theta, \lambda}}\left(z_{0}\right)\right)
$$

is a subcurve of $\partial \exp \left((i \pi / 2) V\left(z_{0}, \lambda\right)\right)$.

The fact that a simple closed curve cannot contain any simple closed curve other than itself yields that $\partial V\left(z_{0}, \lambda\right)$ is given by

$$
(-\pi, \pi] \ni \theta \longmapsto Q_{e^{i \theta}, \lambda}\left(z_{0}\right)
$$

The proof of Lemma 2.2 is complete.

Proof of Theorem 1.5. The proof of Theorem 1.5 follows from Lemmas 1.2, 1.7, and 2.2.

Proof of Theorem 1.8 is a consequence of Theorem A and the following lemma.

Lemma 2.3. For any fixed $a \in \overline{\mathbb{D}}$, let $\phi_{a}: \mathbb{D} \rightarrow \overline{\mathbb{D}}$ be defined by

$$
\begin{aligned}
\phi_{a}(z) & =\frac{\bar{z}-a}{1-a z}, \quad z \in \mathbb{D}, \\
\mathcal{M} & =\left\{\phi_{a}: a \in \overline{\mathbb{D}}\right\},
\end{aligned}
$$

and $V_{0}\left(z_{0}\right)=\left\{\phi_{a}\left(z_{0}\right): \phi_{a} \in \mathcal{M}\right\}$. Then $\mathcal{M} \subset$ 乃 $\mathcal{L}$ and $V_{0}\left(z_{0}\right)=\overline{\mathbb{D}}$.

Proof. We write

$$
\phi_{a}(z)=\frac{\bar{z}-a}{1-a z}=|z|^{2} G(z, a)+H(z, a),
$$

and so, we have $\phi_{\bar{z}}(0)=1$, where

$$
G(z, a)=a \sum_{n=0}^{\infty}(a z)^{n}, \quad H(z, a)=\bar{z}-a \sum_{n=0}^{\infty}(a z)^{n} .
$$

Obviously, $\phi_{a}(z)=(\bar{z}-a) /(1-a z)$ is a biharmonic mapping and $\phi_{a}(\mathbb{D}) \subset \mathbb{D}$, from which the first assertion in Lemma 2.3 follows. 
Since $z_{0} \in \mathbb{D} \backslash\{0\}$, we see that $g_{z_{0}}(w)=\left(\overline{z_{0}}-w\right) /\left(1-z_{0} w\right)$ is a conformal automorphism of the unit disk $\mathbb{D}$ and the range $g_{z_{0}}(\overline{\mathbb{D}})$ is $\overline{\mathbb{D}}$ itself. By hypotheses, we know that for any $q \in \mathcal{M}$, $q\left(z_{0}\right) \in \overline{\mathbb{D}}$. Hence $V_{0}\left(z_{0}\right)$ coincides with $\overline{\mathbb{D}}$.

\section{Acknowledgment}

The research was partly supported by NSFs of China (no. 10771059).

\section{References}

[1] J. Clunie and T. Sheil-Small, "Harmonic univalent functions," Annales Academiae Scientiarum Fennicae. Series A, vol. 9, pp. 3-25, 1984.

[2] P. Duren, Harmonic Mappings in the Plane, vol. 156 of Cambridge Tracts in Mathematics, Cambridge University Press, Cambridge, UK, 2004.

[3] H. Lewy, "On the non-vanishing of the Jacobian in certain one-to-one mappings," Bulletin of the American Mathematical Society, vol. 42, no. 10, pp. 689-692, 1936.

[4] Z. Abdulhadi and Y. Abu Muhanna, "Landau's theorem for biharmonic mappings," Journal of Mathematical Analysis and Applications, vol. 338, no. 1, pp. 705-709, 2008.

[5] Z. AbdulHadi, Y. Abu Muhanna, and S. Khuri, "On univalent solutions of the biharmonic equation," Journal of Inequalities and Applications, no. 5, pp. 469-478, 2005.

[6] Sh. Chen, S. Ponnusamy, and X. Wang, "Landau's theorem for certain biharmonic mappings," Applied Mathematics and Computation, vol. 208, no. 2, pp. 427-433, 2009.

[7] Sh. Chen, S. Ponnusamy, and X. Wang, "Properties of some classes of planar harmonic and planar biharmonic mappings," submitted.

[8] S. A. Khuri, "Biorthogonal series solution of Stokes flow problems in sectorial regions," SIAM Journal on Applied Mathematics, vol. 56, no. 1, pp. 19-39, 1996.

[9] M. Chuaqui, P. Duren, and B. Osgood, "Ellipses, near ellipses, and harmonic Möbius transformations," Proceedings of the American Mathematical Society, vol. 133, no. 9, pp. 2705-2710, 2005.

[10] Ch. Pommerenke, Boundary Behaviour of Conformal Maps, vol. 299 of Grundlehren der Mathematischen Wissenschaften, Springer, Berlin, Germany, 1992.

[11] M. Chuaqui and R. Hernández, "Univalent harmonic mappings and linearly connected domains," Journal of Mathematical Analysis and Applications, vol. 332, no. 2, pp. 1189-1194, 2007.

[12] J. M. Anderson, J. Clunie, and Ch. Pommerenke, "On Bloch functions and normal functions," Journal für die Reine und Angewandte Mathematik, vol. 270, pp. 12-37, 1974.

[13] H. Yanagihara, "Regions of variability for convex functions," Mathematische Nachrichten, vol. 279, no. 15, pp. 1723-1730, 2006.

[14] S. Ponnusamy and A. Vasudevarao, "Region of variability of two subclasses of univalent functions," Journal of Mathematical Analysis and Applications, vol. 332, no. 2, pp. 1323-1334, 2007. 


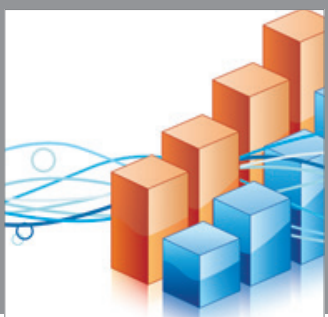

Advances in

Operations Research



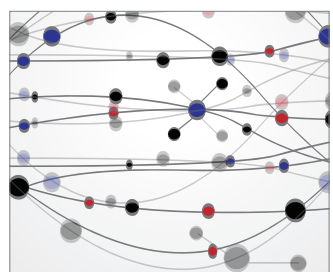

\section{The Scientific} World Journal
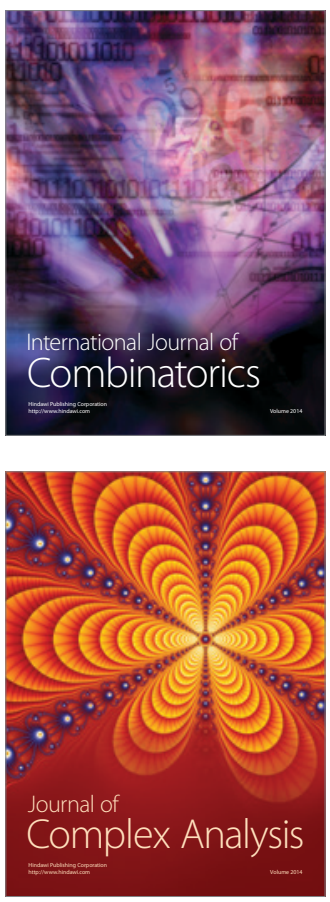

International Journal of

Mathematics and

Mathematical

Sciences
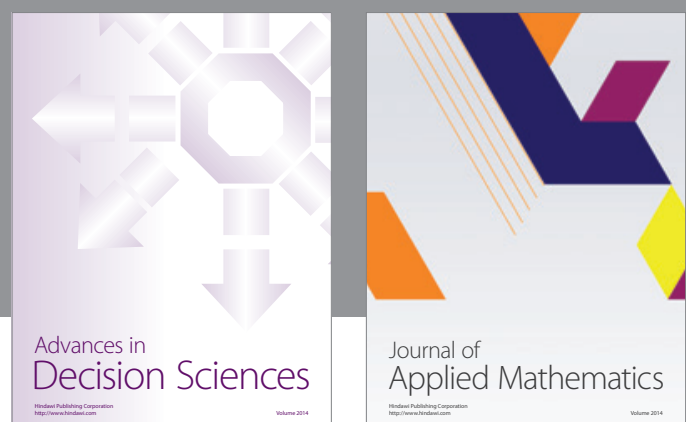

Journal of

Applied Mathematics
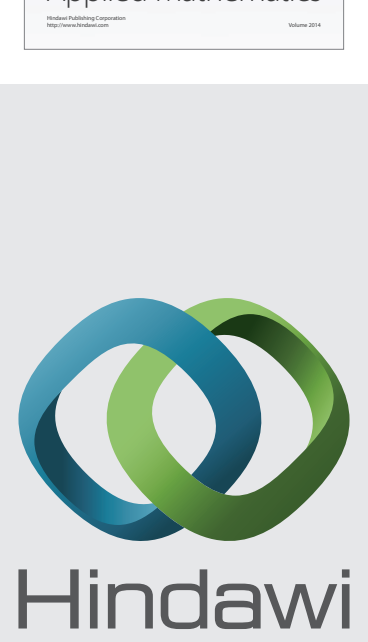

Submit your manuscripts at http://www.hindawi.com


Mathematical Problems in Engineering
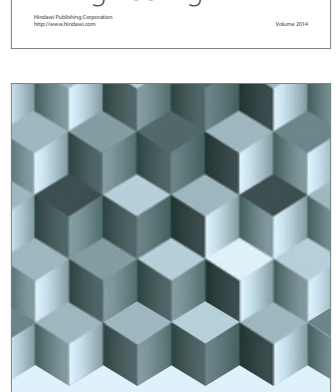

Journal of

Function Spaces
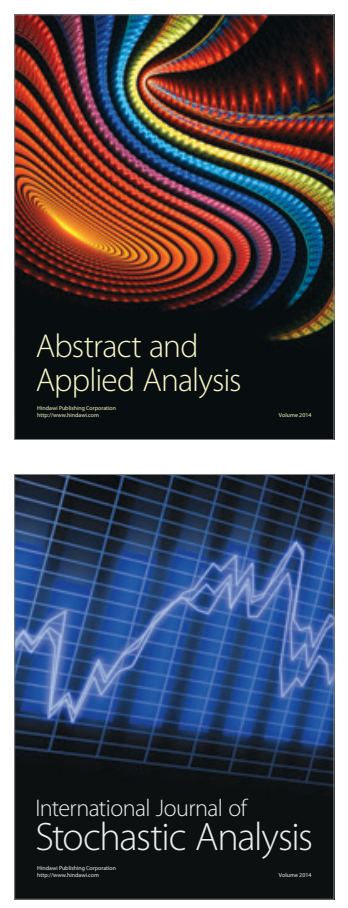

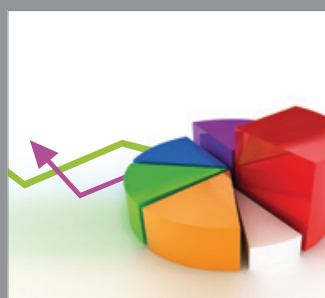

ournal of

Probability and Statistics

Promensencen
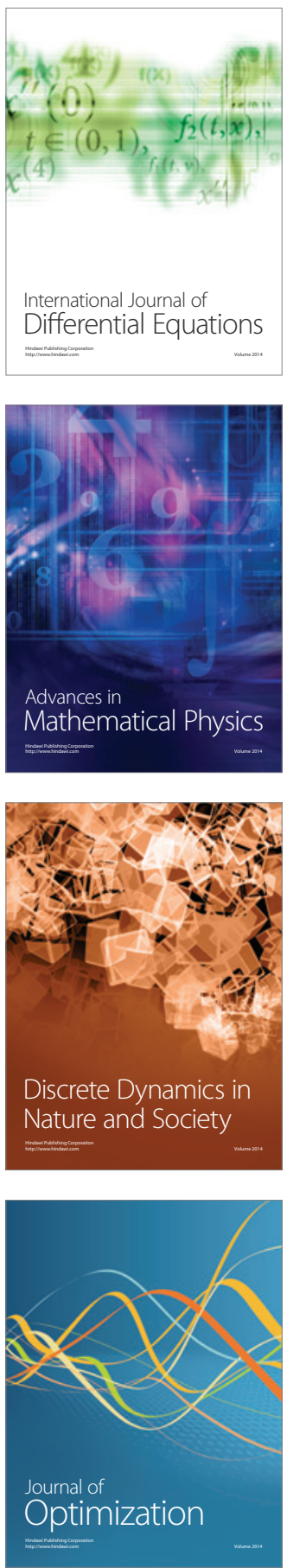